\title{
ENERGY EFFICIENT MAC PROTOCOLS FOR WIRELESS SENSOR NETWORKS: A SURVEY
}

\author{
Bhavana Narain, Anuradha Sharma, Sanjay Kumar and Vinod Patle \\ SoS in CS \& IT, Pt. Ravishankar Shukla University, Raipur, Chhattisgarh, India \\ \{narainbhawna, anuradha.shinestar, sanraipur, patlevinod\}@gmail.com
}

\begin{abstract}
Use of Wireless sensor networks have been widely seen in the fields of target detection and tracking, environmental monitoring, industrial process monitoring, and tactical systems.In wireless sensor networks nodes work with a incomplete power source, energy efficient operations in an important factor of the nodes in wireless sensor network. Energy conservation plays important role in different layers of the TCP/IP protocol suit, and for MAC layer it is the effective part. Therefore, to work in wireless communicating sensors network, we use MAC protocol which improve energy efficiency by increasing sleep duration, decreasing idle listening and overhearing, and eliminating hidden terminal problem or collision of packets. In this paper First section we describe the accessible energy-efficient MAC protocols for sensor networks their energy saving method. In Second section we discuss the architecture of same protocols and then compare same protocols depending on their Advantages and Disadvantages.
\end{abstract}

\section{Keywords}

Wireless sensor Network, Mac protocol, Energy.

\section{INTRODUCTION}

Now a days intrusion detection, defense, climate control, medical systems ,environment monitoring, robotic exploration, smart spaces, disaster management, target tracking, wildlife habitat monitoring, scientific application, are uses the Wireless Sensor Network. The Wireless sensor networks are made up of one or more battery-operated sensor devices with embedded processor, small memory and low power radio. Coverage and communication range for sensor nodes compared to other mobile devices is limited due to low power capacities of sensor nodes. Sensor networks are composed of large number of nodes to cover the target area. Nodes in wireless sensor network communicate with each other to give a common task [1].

How to efficiently utilize the limited amount of energy has been the primary concern in designing MAC protocols for WSNs [2]. As there was a challenge for WSN designers is to develop a system that will run for years, they used not only robust hardware and software, but also lasting energy sources.

In shared channel to manage the access of active node, Medium access control schemes are used .MAC protocol provides following functional it: 
- Framing: Define the frame format and perform data encapsulation and de encapsulation for communication between devices.

- Medium access: It controls the devices to participate in communication at any time. Medium access becomes a main function of wireless MAC protocols since it broadcasts easily which cause data corruption through collisions.

- Reliability: It ensure successful transmission between devices. Mostly through acknowledgement (ACK) messages and retransmissions when necessary.

- Flow control: From beginning to end prevent frame loss, overloaded recipient buffers.

- Error control: In frames delivered to upper layers, to have power over the amount of errors present it uses error detection or error correction codes [3].

\section{AtTributes of Good MAC PRotocol}

In the wireless sensor networks, for designing high-quality MAC protocol, these attributes are to be measured [4].

- Energy Efficiency: Energy efficiency are the first attribute. Battery powered consist in The sensor nodes and it is often extremely complicated to change or recharge batteries for these sensor nodes. Sometimes it is helpful to replace the sensor node rather than recharging them.

- Latency: The second is latency. Latency requirement basically depends on the application. the detected events must be reported to the sink node in real time In the sensor network applications, so that the suitable action could be taken immediately.

- Throughput: With different applications the throughput requirement also varies. A few sensor network application require to sample the information with fine temporal resolution. In such sensor applications it is better that sink node receives more data.

- Fairness: In several sensor network applications when bandwidth is limited, it is compulsory to confirm that the sink node receives information from all sensor nodes fairly. However along with all of the above aspects the energy efficiency and throughput are the key aspects. By minimizing the energy wastage energy efficiency can be increased [4].

\section{ENERGY WASTE IN MAC PROTOCL}

The reason of wastage of energy in a MAC protocol for wireless sensor networks are the following [5].

$>$ Collision: - Some time the packet gets corrupted during transmission these packet need to be discarded and resent, these lead to increased energy consumption.

$>$ Control Packet Overhead:- Energy is also required for Sending and receiving control packets due to this less useful data packets can be transmitted. 
$>$ Idle Listening: - Extra energy is also consumed for Listening to receive possible traffic which is not sent.

$>$ Overhearing:- Sometime nodes can pickup which are destined to other nodes. These also leads to unnecessary consume of energy.

Reducing the energy wasted idle listing protocols like SMAC, TMAC and CMAC can be used. SMAC Traditional wakeup scheduling approach which uses fixed duty cycle [4].

\section{Duty Cycle =Listen Interval/ Frame Length}

SMAC and TMAC reduce energy consumption by using Coordinated scheduling, but this requires periodic synchronization. CMAC supports low latency and avoids synchronization overhead [6]. CMAC allows operation at very low duty cycles by using unsynchronized sleep scheduling .TMAC uses adaptive duty cycle and has the advantage of dynamically ending active part [1].

\section{TAXONOMY OF MAC PROTOCOLS}

The medium access control protocols can be broadly divided into two categories.

1) Schedule based.

2) Contention based.

The schedule based protocols is based on strict time synchronization requirements. They schedule transmit \& listen periods, thus avoiding collisions, overhearing and idle listening. These protocols are based on Carrier Sense Multiple Access (CSMA) technique, they have higher costs for message collisions, overhearing and idle listening [5].The contention based protocols relax time synchronization requirements and can easily adjust to the topology changes by joining some new nodes. Others nodes may die few years after deployment.

\subsection{SENSOR MAC (S-MAC)}

In wireless sensor network, the Sensor S-MAC protocol is a contention based MAC protocol. It is an improved version of IEEE 802.11 protocol .The sensor node periodically goes to the fixed sleep cycle for the medium access control protocol. the time frame is divided into to part: one for a listening session and the other for a sleeping session In SMAC,. the sensor node are capable to communicate with additional nodes and send some control packets such as SYNC, RTS (Request to Send), CTS (Clear to Send) and ACK(Acknowledgement) are only for listen period. By a SYNC packet exchange all nearest nodes can synchronize collectively and using RTS/CTS switch over the two nodes can communicate with each other. the Fig. 1 discribe the basic snode scheme where node 1 transmits data to node 2 is shown . even IF there is no reception/transmission A lot of energy is still wasted in this protocol during listen period as the sensor will be awake [7].

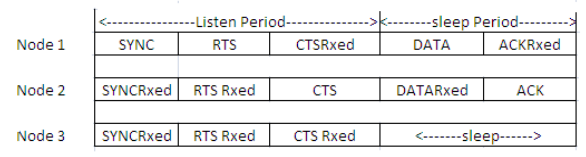

Figure 1: Basic S-MAC Scheme, Node 1 Transmits Data to Node 2 


\subsection{BERKELEY MAC (BMAC)}

Another contention based MAC protocol is Berkeley Media Access Control (B-MAC) which is widely used in WSNs [8]. B-MAC is like to Aloha with Preamble Sampling [9], BMAC duty cycles the radio transceiver i.e. the Sensor node turns ON/OFF again and again without missing the data packets. The preamble length is provided as a parameter to the upper layer, which provides optimal trade-off between energy savings and latency or throughput [5]. BMAC is also similar to CSMA protocol with having a feature of Low Power Consumption [8]. Unsynchronized duty cycling and long preambles are used in BMAC to wake up receivers. BMAC increase reliability and channel assessment by a filter mechanism. The sensor node can change any operating variables in the protocol, such as back off values. This provides a flexibility interface. BMAC employs an adaptive preamble sampling scheme which minimize idle listening and reduce duty cycle as shown in fig.2. B-MAC duty cycles the radio through. Periodic channel sampling that are called Low Power Listening (LPL). The clear channel assessment (CCA) technique is used by BMAC to decide if a packet is arriving when node wakes up. If no packet arrived timeout puts node back to sleep. CCA and packet bakeoffs are used by BMAC for channel arbitration, link layer acknowledgments for reliability. There are no synchronization, RTS, CTS in BMAC [1].

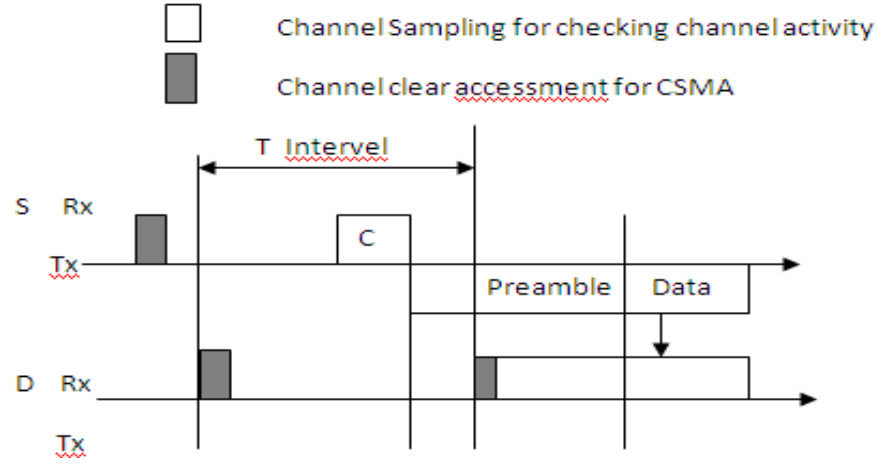

Figure 2. Preamble sampling in BMAC

\subsection{TIMEOUT MAC (TMAC) / DYNAMIC SENSOR MAC (DSMAC)}

Static sleep-listen periods of S-MAC result in high latency and lower throughput as indicated earlier. Timeout- MAC (T-MAC) [9] is proposed to enhance the poor results of S-MAC protocol under variable traffic load. In T-MAC, when no activation event has occurred listen period ends for a time threshold TA. Along with some solutions the decision for TA is presented to the early sleeping problem defined in [9]. Dynamic Sensor-MAC (DSMAC) [10] it sum the dynamic duty cycle feature to S-MAC. The aim is to Decrease the latency for delaysensitive applications. Within the SYNC period, all nodes share their one-hop latency values (time between the reception of a packet into the queue and its transmission). All nodes start with the same duty cycle. Fig.3 conceptually depicts DSMAC duty cycle doubling. When a receiver node notices that average one-hop latency value is high, it decides to shorten its sleep time and announces it within SYNC period. Accordingly, after a sender node receives this sleep period decrement signal, it checks its queue for packets destined to that receiver node. If there is one, it decides to double its duty cycle when its battery level is above a specified threshold. 
The duty cycle is doubled so that the schedules of the neighbors will not be affected. The latency observed with DSMAC is better than the one observed with S-MAC. Moreover, it is also shown to have better average power consumption per packet [3].

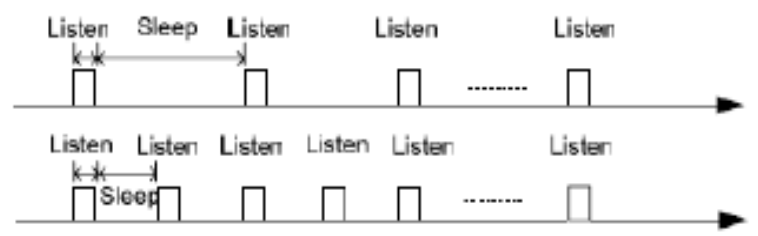

Figure 3 DSMAC duty cycle doubling

\subsection{WISE MAC (WMAC)}

The working of WiseMAC [11] when the sender starts the preamble before the receiver is expected to wake up rather than selecting a random time. For alerting the receiving node the preamble precedes each data packet. The nodes which are presents in the network sample is having the medium with a common period, but their relative schedule offsets are independent. If a node finds the medium busy after it wakes up and samples the medium, it regularly listen till it receives a data packet or the medium comes to the idle state. The size of the preamble in WiseMAC is initially set to be equal to the sampling period. Fig.4 shows preamble minimization in WiseMAC. In the Process of learning and refreshing their neighbor's the nodes sleep schedule during every data exchange as part of the Acknowledgment message. The node keeps a table of the sleep schedules of its neighbors and decides own schedule accordingly. The possibility to decrease the collisions caused by that specific start time of a wake-up preamble, a random wake-up preamble can be adopted. The wake-up preamble length gets affects by the clock drifts between the source and the destination [1].

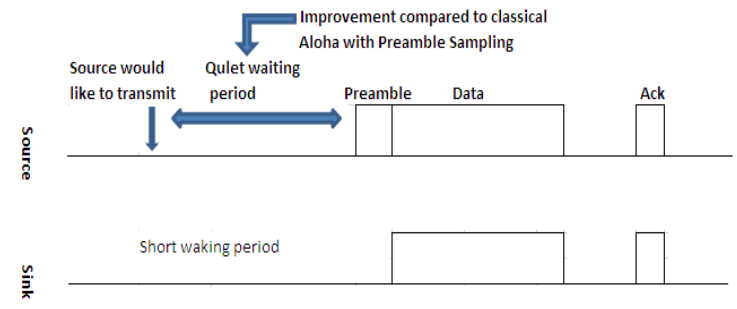

Figure 4 WiseMAC preamble minimization

\subsection{TRAFFIC-ADAPTIVE MAC PROTOCOL (TRAMA)}

TRAMA [14] is algorithm based on a TDMA it proposed to increase the utilization of classical TDMA in an energy efficient manner. It is like Node Activation Multiple Access (NAMA) [15], where a distributed election algorithm is used to select one transmitter within two-hop neighborhood for each time slot. 
Random-access and Scheduled access (transmission) periods are two part of time. For establish two-hop topology information Random-access period is used . It is assumed that Mac layer can calculate the transmission duration needed by the information passed by the application layer. This transmission duration is denoted as SCHEDULE_INTERVAL. Then at time t, the node calculates the number of slots for which it will have the highest priority among two-hop neighbors within the Period [tt+ SCHEDULE_INTERVAL]. The node announces the slots which will use as well as the intended receivers for these slots with a schedule packet. A bitmap whose length is equal to the number of its neighbors is used to indicates the intended receivers by the scheduled packets. Bits correspond to one-hop neighbors ordered by their identities. identities of the potential senders receiver, one hop neighbors are evaluated for re-use of those slots [3].

\subsection{DATA GATHERING MAC (DMAC)}

The another schedule based MAC protocol is Data-Gathering Medium Access Control (DMAC) [16]. Which has been planned and optimized for tree based data gathering in wireless sensor network. The low latency and still maintaining the energy efficiency is the main aim of this MAC protocol. In this the time is divided in small slots and runs carrier sensing multiple access (CSMA) with acknowledgement within each slot to transmit/receive one packet. The sensor node sometimes executes the basic sequence of ' 1 ' transmit, ' 1 ' receive and ' $n$ ' sleep slots. In this approach a single packet from a source node at depth ' $k$ ' in the tree reaches the sink node with a delay of just ' $\mathrm{k}$ ' time slots. This delay is very small and it is in the order of tens of milliseconds. A data gathering (converge cast) tree with staggered DMAC slots is shown in Fig.5. D-MAC includes an overflow mechanism to handle the problem when each single source node has low traffic rate but the aggregate rate at intermediate node is larger than the basic duty cycle. In this mechanism after forwarding the packet, the sensor node will remain awake for one extra time slot.

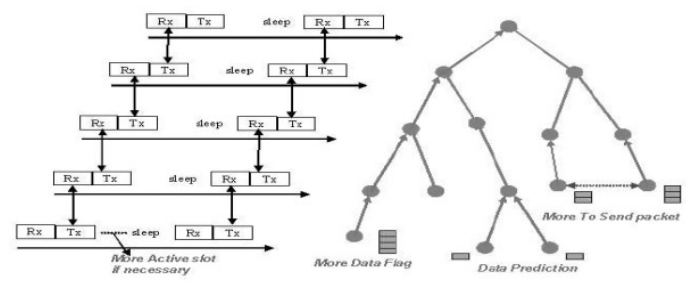

Figure 5: Data gathering tree in D-MAC scheme

thus, if two children were differing for parents accept slot, the loosing child will get a second opportunity to send its packet. The D-MAC uses a separate control packet named MTS (More to Send) to solve the difficulty of the interference between nodes on the dissimilar branches of the tree. The MTS packet makes all the nodes on the multi-hop path to remain active in case of nodes failure due to interference. in terms of energy efficiency, latency and throughput in both multi-hop chain topology and random data gathering tree topology The simulation results shows that the D-MAC protocol outperforms the Sensor S-MAC protocol [5]. 


\subsection{CONVERGENT MAC (CMAC)}

CMAC [6] is a novel MAC layer protocol; it improves energy efficiency and the latency by utilizing aggressive RTS, Anycast and convergent packet forwarding mechanisms. It uses "aggressive RTS" equipped with double channel check for channel assessment as shown in fig.6. In CMAC there is unsynchronized sleep scheduling (or duty cycling) when there is no packet to transmit. it avoids synchronization overhead during supporting low latency. when there is no traffic, it uses zero communication CMAC allows operation at very low duty cycles. In the situation of traffic, CMAC first uses anycast for packet forwarding to wake up forwarding nodes or to quickly discover forwarder and then converges from route- suboptimal anycast with unsynchronized duty cycling to route-optimal unicast with synchronized scheduling. For flow initialization it use anycast and for flow stabilization it uses convergent Packet Forwarding.

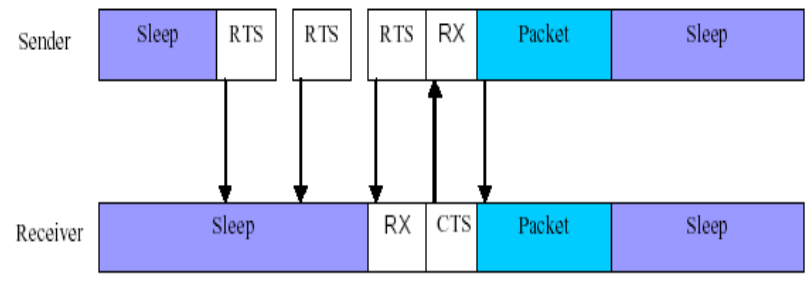

Figure 6. Aggressive RTS in Convergent MAC

the checking of the channel twice to avoid missing activities, time between the two checks should be larger than inter-RTS separation and smaller than RTS duration as shown in fig 7.Receiver needs to check if co-ordination that channel is busy after waking up. Time between the two checks should be larger than inter-RTS separation and should be smaller than RTS duration [1].

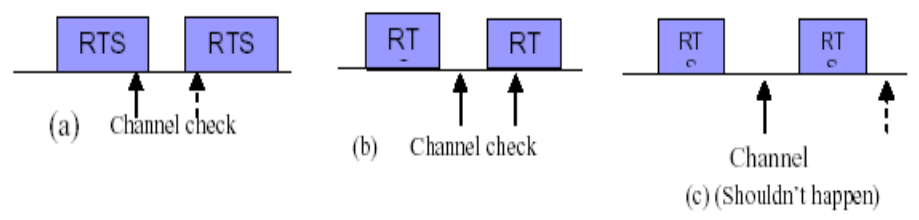

Figure 7. Double channel checking in Convergent MAC

\section{RELATED WORK}

By [4] Contention-based and TDMA protocols are the main component of the MAC design for wireless sensor networks. When nodes are in idle mode the energy consumption using this MAC is very high.

By [17] Mac protocol work for the CSMA and tonebased MANET.

By [18] WSN MAC protocol focus mainly on energy efficiency. 
International Journal of Computer Science \& Engineering Survey (IJCSES) Vol.2, No.3, August 2011

By [19] the recent work on MAC protocol design in sensor networks [1, 6, 7] focused on energy efficiency and coordination instead of fairness, delay, and bandwidth utilization.

\section{COMPARATIVE STUDY OF EXISTING ENERGY EFFICIENT MAC PROTOCOLS}

Table 1 compares the different MAC protocols discussed in the preceding section. Here, we have shown their comparison by taking parameter scheme used, energy saving, advantages, disadvantages [1].

Table-1: Comparative study of Energy-efficient MAC protocols.

\begin{tabular}{|c|c|c|c|c|}
\hline $\begin{array}{l}\text { Name of } \\
\text { protocol }\end{array}$ & Scheme used & Energy Saving & Advantages & Disadvantages \\
\hline SMAC & $\begin{array}{l}\text { Fixed duty } \\
\text { cycle, virtual } \\
\text { cluster, CSMA }\end{array}$ & $\begin{array}{l}\text { Power savings } \\
\text { over standard } \\
\text { CSMA/CAMAC }\end{array}$ & $\begin{array}{l}\text { Low energy } \\
\text { consumption } \\
\text { when traffic is low }\end{array}$ & $\begin{array}{l}\text { Sleep latency, } \\
\text { problem with } \\
\text { Broadcast }\end{array}$ \\
\hline BMAC & $\begin{array}{l}\text { LPL, channel } \\
\text { assessment } \\
\text { software } \\
\text { interface }\end{array}$ & $\begin{array}{l}\text { Better power } \\
\text { savings, latency, } \\
\text { and throughput } \\
\text { than S-MAC }\end{array}$ & $\begin{array}{l}\text { Low overhead when } \\
\text { network is idle, } \\
\text { imple to implement } \\
\text { Consumes less } \\
\text { power }\end{array}$ & $\begin{array}{l}\text { Overhearing, bad } \\
\text { performance at } \\
\text { heavy traffic. Long } \\
\text { transmission latency }\end{array}$ \\
\hline TMAC & $\begin{array}{l}\text { Adaptive duty } \\
\text { cycle, } \\
\text { overhearing, } \\
\text { FRTS }\end{array}$ & $\begin{array}{l}\text { Uses } 20 \% \text { of } \\
\text { energy used in } \\
\text { S-MAC. }\end{array}$ & $\begin{array}{l}\text { Adaptive active } \\
\text { time }\end{array}$ & $\begin{array}{l}\text { Early sleeping } \\
\text { problem }\end{array}$ \\
\hline $\begin{array}{l}\text { WISE } \\
\text { MAC }\end{array}$ & $\begin{array}{l}\text { Minimized } \\
\text { preamble } \\
\text { sampling, } \\
\text { schedule }\end{array}$ & $\begin{array}{l}\text { Better than } \\
\text { SMAC and Low } \\
\text { Power Listening }\end{array}$ & $\begin{array}{l}\text { Energy } \\
\text { Consumption both } \\
\text { at sender And } \\
\text { receiver, and at non } \\
\text { target receiver, } \\
\text { increase latency at } \\
\text { each hop. }\end{array}$ & $\begin{array}{l}\text { Low power for low } \\
\text { traffic, Do not incur } \\
\text { overhead due to } \\
\text { synchronization. }\end{array}$ \\
\hline TRAMA & TDMA & $\begin{array}{l}\text { Utilization of } \\
\text { classical TDMA }\end{array}$ & $\begin{array}{l}\text { Higher energy } \\
\text { efficiency \& } \\
\text { throughput }\end{array}$ & $\begin{array}{l}\text { time is divided into } \\
\text { random access period }\end{array}$ \\
\hline DMAC & $\begin{array}{l}\text { Converge cast } \\
\text { communication }\end{array}$ & Low latency & $\begin{array}{l}\text { Energy saving and } \\
\text { low letency }\end{array}$ & $\begin{array}{l}\text { Aggregate rate is } \\
\text { larger }\end{array}$ \\
\hline
\end{tabular}


International Journal of Computer Science \& Engineering Survey (IJCSES) Vol.2, No.3, August 2011

\begin{tabular}{|l|l|l|l|l|}
\hline CMAC & $\begin{array}{l}\text { Aggressive } \\
\text { Ack. Anycast. } \\
\text { convergent } \\
\text { packet } \\
\text { forwarding }\end{array}$ & $\begin{array}{l}\text { consumes less } \\
\text { energy than } \\
\text { existing } \\
\text { solutions }\end{array}$ & $\begin{array}{l}\text { high throughput, } \\
\text { low latency \& } \\
\text { consumes less } \\
\text { energy }\end{array}$ & Not Yet found \\
& & & \\
\hline
\end{tabular}

\section{CONSLUSION \& FUTURE WORK}

Designing a MAC protocol which can improve energy-efficiency to extend network lifetime in wireless sensor networks is a challenging problem. It is mainly due to stringent resource constraint both in sensor nodes and in wireless media. Several energy-efficient medium access control protocols both contention-based and Schedule-based for the wireless sensor network that have been proposed by the researchers are presented in this paper.

Although there are various MAC layer protocols proposed for sensor networks, there is no protocol accepted as a standard. One of the reasons behind this is the MAC protocol choice will, in general, be application-dependent, which means that there will not be one standard MAC for WSNs.

\section{REFERENCES}

[1] A Roy and N Sarma, "Energy Saving in MAC Layer of Wireless Sensor Networks:a Survey" ( 2010) Department of Computer Science and Engineering, Tezpur University, INDIA ,alakroy@yahoo.co.in, nitya@tezu.ernet.in "National Workshop in Design and Analysis of Algorithm (NWDAA)", Tezpur University, India".

[2] Kurtis Kred, and Prasant Mohapatra, "Medium access control in wireless Sensor networks", ( 31 July 2006) Conference on Computer Networks, vol. 51, pp. 961- 994.

[3] Isha Batra, Dr. Trilok C. Aseri" Comparison of Efficient MAC Protocols for Wireless SensorNetworks" (6 February 2010) Department of Computer Science \& Engineering, PEC University of Technology, Sector-12, Chandigarh-160012, India 1 ishabatraladwa@gmail.com,2 a_trilok_chand@yahoo.com Proceedings of the Int. Conf. on Information Science and Applications ICISA 2010 , Chennai, India.

[4] Wei Ye, J.Heidemann and D. Estrin: "An Energy- Efficient MAC Protocol for Wireless Sensor Networks", (June 2002).IEEE INFOCOM, New York, Vol. 2, pp. 1567-1576.

[5] Tijs van Dam and Koen Langendoen.” An adaptive energy-efficient mac protocol for wireless sensor networks", (November 2003) In Proceedings of the First ACM SenSys Conference, pages 171180, Los Angeles, California, USA, ACM.

[6] Sha Liu, Kai-Wei Fan and Prasun Sinha , "An Energy Efficient MAC Layer Protocol Using Convergent Packet Forwarding for Wireless Sensor Networks", (2007) IEEE SECON.

[7] Rajesh Yadav Electronis and Radar Development Establishment Defense R \& D Organization, Bangalore, India Shirshu Varma Indian Institute of Information Technolgy, Allahabad, India N. Malaviya Institute of Engineering \& Technology, Lucknow, India "A SURVEY OF MAC PROTOCOLS FOR WIRELESS SENSOR NETWORKS". 
International Journal of Computer Science \& Engineering Survey (IJCSES) Vol.2, No.3, August 2011

[8] J. Polastre, J. Hill, and D. Culler. "Versatile low power media access for wireless sensor networks", ( November 2004) In The Second ACM Conference on Embedded Networked Sensor Systems (SenSys), pages 95-107.

[9] T.V. Dam and K. Langendoen, "An Adaptive Energy-Efficient MAC Protocol for Wireless Sensor Networks", (November 2003) The First ACM Conference on Embedded Networked Sensor Systems (Sensys'03), Los Angeles, CA, USA.

[10] P. Lin, C. Qiao, and X. Wang, "Medium access control with a dynamic duty cycle for sensor networks", ( March 2004) IEEE Wireless Communications and Networking Conference, Volume: 3, Pages: 1534 - 1539, 21-25.

[11] A. El-Hoiydi: "Aloha with Preamble Sampling for Sporadic Traffic in Ad-hoc Wireless Sensor Networks", (April 2002)in Proceedings of IEEE International Conference on Communications .

[12] C.C. Enz, A. El-Hoiydi, J.-D. Decotignie, V. Peiris: ” WiseNET: An Ultralow-Power Wireless Sensor Network Solution", (August 2004) IEEE Computer, Vol. 37, Issue 8.

[13] A. El-Hoiydi: "Spatial TDMA and CSMA with Preamble Sampling for Low Power Ad-hoc Wireless Sensor Network“", (July 2002) Proceedings of ISCC '02, Seventh International Symposium on Computers and Communications, pp. 685-692.

[14] V. Rajendran, K. Obraczka, J.J. Garcia-Luna-Aceves, "Energy- Efficient, Collision-Free Medium Access Control for Wireless Sensor Networks",( 5-7 November 2003) Proc. ACM SenSys 03, Pages:181 - 192, Los Angeles, California.

[15] L. Bao and J.J. Garcia-Luna-Aceves, "A New Approach To Channel Access Scheduling For Ad Hoc Networks"(2001), Seventh Annual International Conference on Mobile Computing and Networking, pp. 210-221.

[16] G. Lu, B. Krishnamachari, C. Raghavendra: "An Adaptive Energy Efficient and Low Latency MAC for Data Gathering in Wireless Sensor Networks" (April 2004), Proceedings of 18thth International Parallel and Distributed Processing Symposium.

[17] Ying Li1, Minglu Li1, Wei Shu2 Min-You Wu1 "FFT-DMAC: A Tone Based MAC Protocol with Directional Antennas" (2007) IEEE GLOBECOM Department of Computer Science and Engineering Shanghai Jiao Tong University Department of Electrical and Computer Engineering The University of New Mexico.

[18] Muneeb Ali, Tashfeen Suleman, and Zartash Afzal Uzmi "MMAC: A Mobility-Adaptive, Collision-Free MAC Protocol for Wireless Sensor Networks"( 2005) Computer Science Department, LUMS \{muneeb,tashfeens,zartash\} @lums.edu.pk 0-7803-8991-3/05 \$20.00 @ IEEE. E. Egea-Lo’ pez Æ J. Vales-Alonso Æ A. S. Martı’nez-Sala Æ J. Garcı’a-Haro Æ

P. Pavo“ n-Mariño Æ M. V. Bueno Delgado “A wireless sensor networks MAC protocol for real-time applications"( 29 June 2006) Springer-Verlag London Limited. 


\section{AUTHORS}

1) Mrs. Bhavna Narain MCA, M.Phil (CS), has been working in the field of computers since last ten years, Currently working in SoS of computer science \& IT.Pt. Ravishankar Shukla University, Raipur, Chhattisgarh, India. Specialization in computer networks(adhoc,mesh).

2) Ms. Anuradha Sharma has done MCA. Currently, she is doing M.Phil (CS \& IT) From SoS of computer science \& IT.Pt. Ravishankar Shukla University, Raipur, Chhattisgarh, India.

3) Dr.Sanjay Kumar has done B.E. (Electrical),M.E.(Computer Science and Engineering) and Ph.D. in Computer Science and Engineering. At present he is Reader in computer Science Department of Pt. Ravishankar Shukla University, Raipur, Chhattisgarh, India. Computer Networking and Paralal Computing are area of his interest.

4) Dr. Vinod Kumar Patle, is done M.Sc. M.C.A and Ph.D. Currently he working as Sr. Lecturer in Computer Science at School of Studies in Computer Science \& I.T., Pt. Ravishankar Shukla University, Raipur (C.G.). His current research is focused on Wireless networking and Sensor Data Mining.
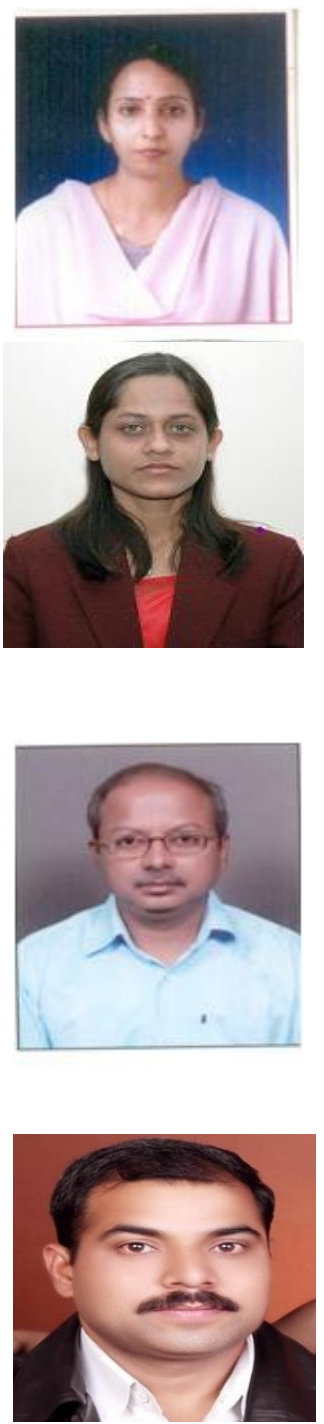